\title{
The Golden Age of Cataclysmic Variables and Related Objects V Concluding Remarks IV
}

\author{
René Hudec ${ }^{1,2,3 *}$ \\ ${ }^{1}$ Astronomical Institute of the Academy of Sciences of the Czech Republic \\ Fricova 298 - CZ 25165 Ondrejov, Czech Republic \\ ${ }^{2}$ Czech Technical University in Prague, Faculty of Electrical Engineering, \\ Technicka 2, CZ 16000 Prague, Czech Republic \\ ${ }^{3}$ Kazan Federal University, Kazan, Russian Federation \\ E-mail: rene.hudec@gmail.com
}

\begin{abstract}
The cataclysmic variables continue to represent important part of modern astrophysics, as demonstrated, already fifth time, by the conference Golden Age of Cataclysmic Variables and Related Objects V held on Mondello (Palermo) in September 2019.
\end{abstract}

The Golden Age of Cataclysmic Variables and Related Objects V (GOLDEN2019)

2-7 September 2019

Palermo, Italy

* Speaker.

${ }^{\dagger}$ A footnote may follow. 


\section{Introduction}

Cataclysmic Variables (CVs) represents in many aspects true astrophysical laboratory where various physical processes and models may be observed, investigated, and tested. They even may play a significant role in the generation of galactic X-ray background, a consequence of surprisingly large fraction of CVs in hard X-ray sources detected by the ESA satellite INTEGRAL. Despite of large theoretical and experimental progress in last years there are still numerous important questions to be answered. Even for well known and studied objects such as SS Cyg or AE Aqr, there are still different opinions in the astronomical community about their nature and involved physical processes. The field is quite complex and interdisciplinary, and only such complex approach has a chance to deliver answers to our questions.

Many important questions regarding investigation of $\mathrm{CVs}$ and related objects were addressed in Palermo, not only during scientific sessions, but also during numerous discussions among participants. There were plenty of opportunities for such discussions during coffee breaks, breakfasts, lunches and dinners, and also during various social activities, all this enjoying the pleasant atmosphere of Sicily (including delicious food, coffee, and wine).

\section{Few subjective comments}

The cataclysmic variables conference in Palermo represents, in my opinion, another successful workshop confirming Prof. Franco Giovannelli/Vulcano theorem (adopted from famous Vulcano Frascati workshops), namely that one can make serious science even keeping smiling. As already confirmed before by long series of Vulcano Frascati (and analogous) workshops, the key for productive workshop is as follows: nice environment, dining and staying together, enough (and good) wine (or beer in countries like Czech Republic). And great participants and speakers, of course, and last but not least experienced conference organizers. There was really wide international participation including countries we only rarely meet on conferences. There was participation of both field leaders as well as young scientists in our conference - many of them have got the possibility to meet the famous professors and senior scientists they know from the literature for the first time. Personally, I have much appreciated the very high quality of most of invited and review talks. In my opinion, this is not trivial as we face the problem of declining quality of such talks at numerous other conferences.

There were nice carefully prepared reviews by various key speakers covering both theory as well as experiment. These lectures have illustrated and demonstrated the great progress both in theory as well as observation/experiment of CVs and related objects over last years. In addition to that, numerous new and mostly detailed observations on particular CVs were presented and discussed, mostly by young scientific fellows.

Common conclusion of many speakers was as follows: we need more and better data, as well as better statistics. Prospects of some of the future experiments/projects still need to be better formulated. In general, there is a need for better monitoring, multi-lambda observations, and in this direction Robotic Telescopes and novel space experiments may help, as proposed and discussed during the workshop. 
What is new if compared with 2011,2013, 2015 and 2017 workshops: Increasing number of young participants and young speakers. I know this is not common in international conferences where it is very difficult for young people to get oral talk. Increasing number of oversee participants (especially but not only, Japan and S. Africa) Very high quality of talks of young speakers Increasing number of female participants and speakers.

In my opinion, the following selected pictures taken during the conference will better illustrate the scope, atmosphere, and success of the conference The Golden Age of Cataclysmic Variables and Related Objects V better than any more words.

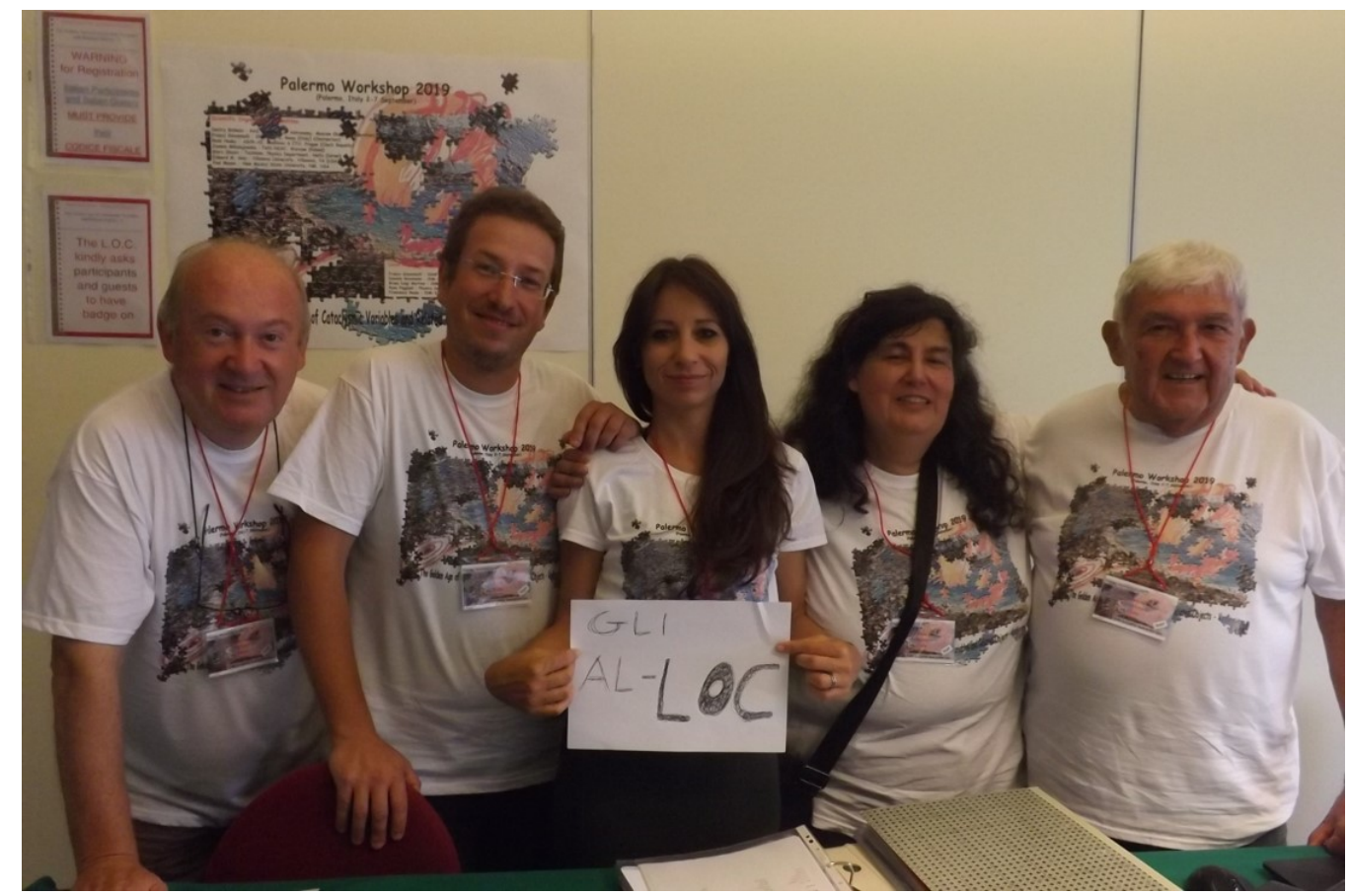

Figure 1: LOC of the conference.

\section{Conclusions}

In conclusion, I am confident that the goal of the workshop was achieved by the synergy between CVs data from ground-based and space-based experiments as well as results from theoretical work. This work on the forefront of the CVs research is likely to result in high-impact scientific research. Hence, the main purpose of the workshop (namely, to discuss in a unique and collaborative setting a broad range of topics in modern CVs astrophysics) had been achieved. I also believe it will encourage collaborative efforts across various topical areas of $\mathrm{CVs}$ research.

\section{Acknowledgements}

The author acknowledges continuous support by the Astronomical Institute of the Czech Academy of Sciences in Ondrejov under Institutional project RVO 67985815 as well as grants GA CR 13-33324S and Mobility MSMT CZ-A project 7AMB18AT036. We also acknowledge the 


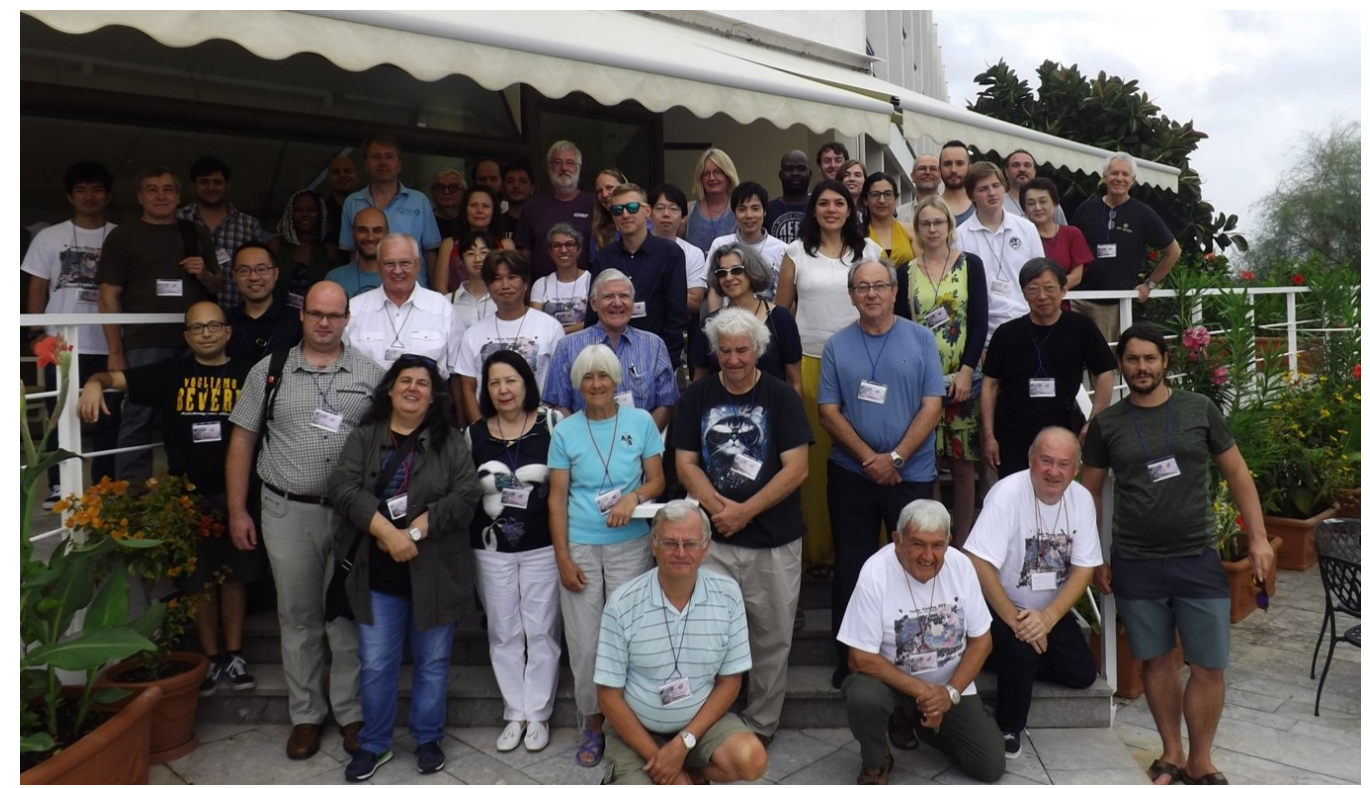

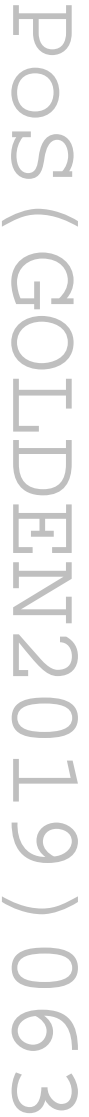

Figure 3: Conference session.

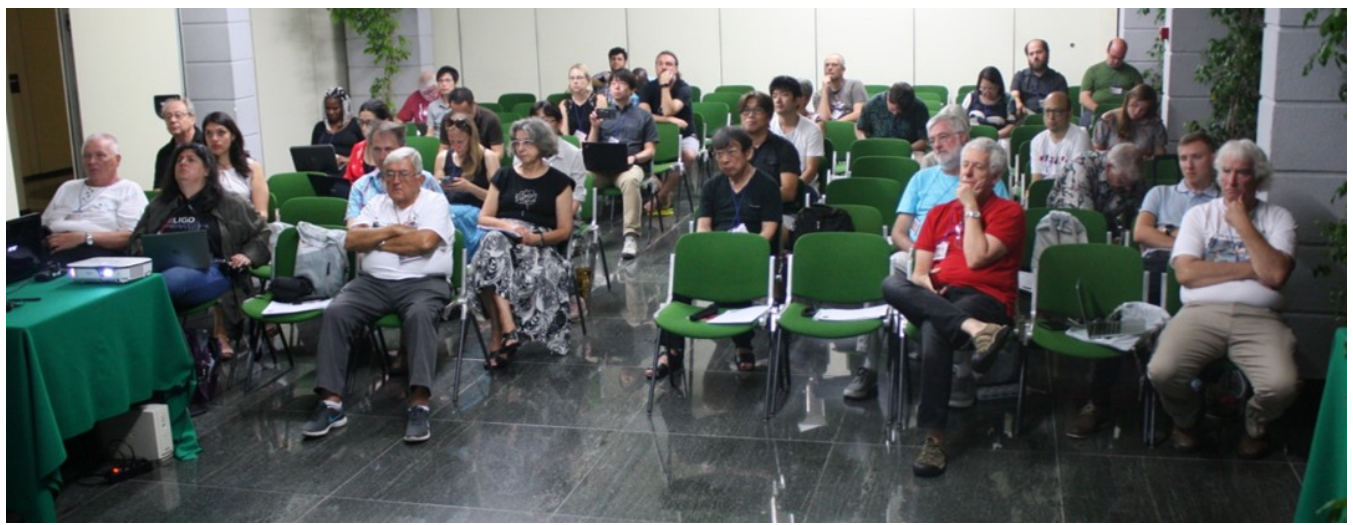

Figure 4: Conference session 2. 


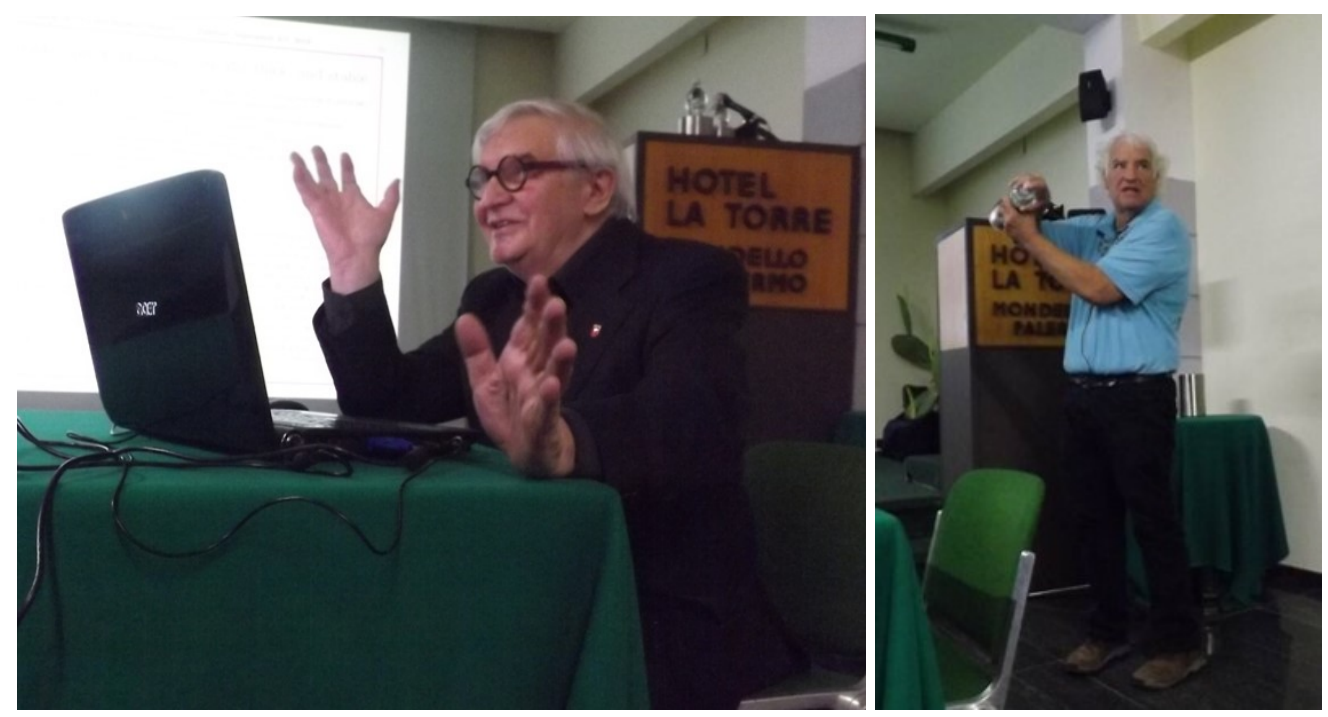

Figure 5: Speakers.

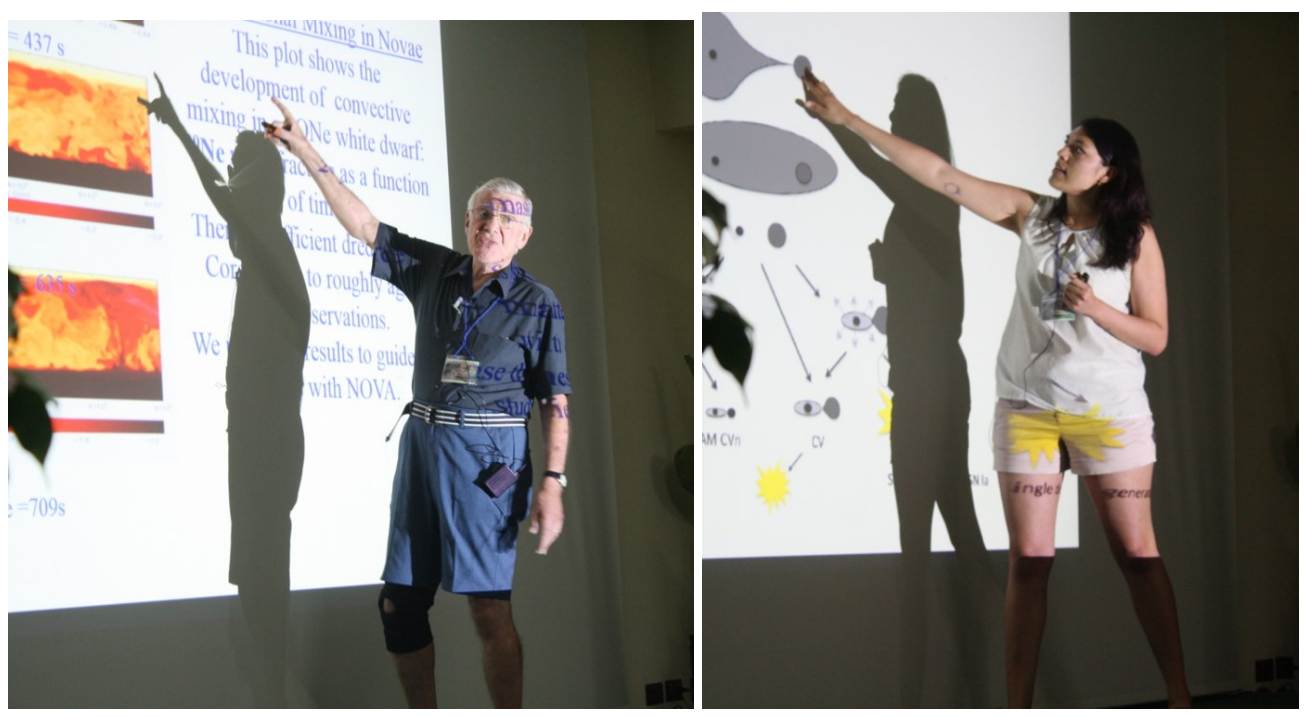

Figure 6: Speakers.

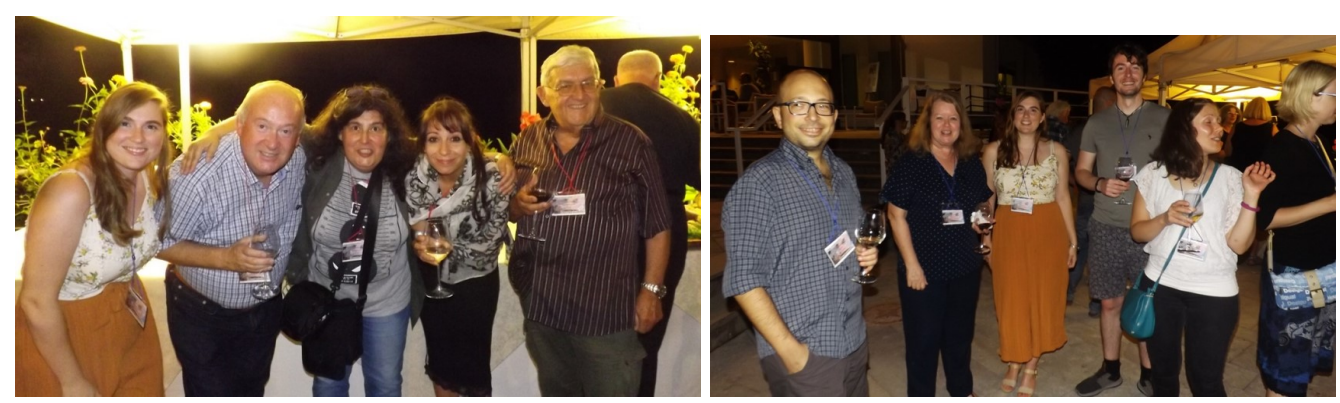

Figure 7: Etruscan wine party. 


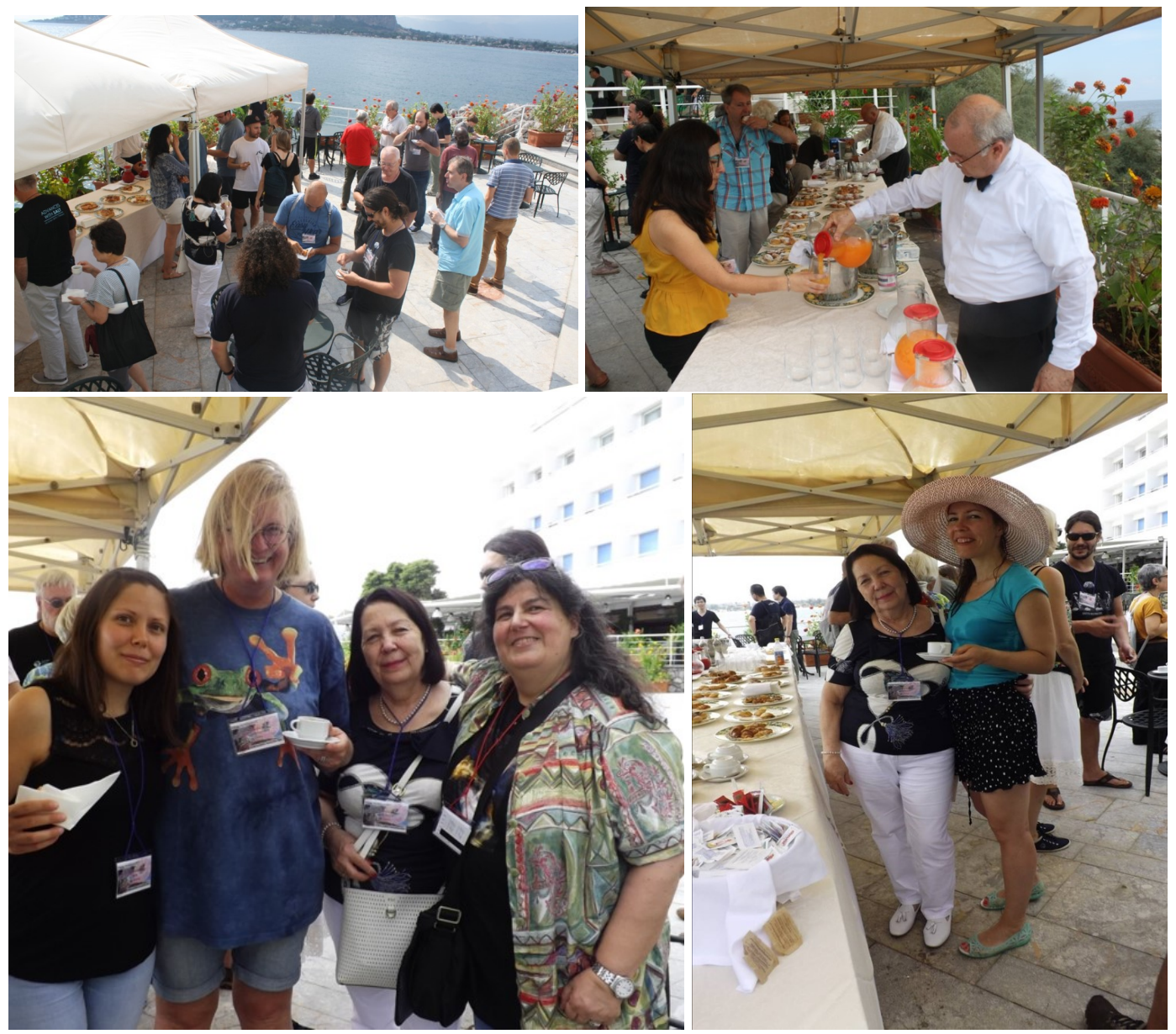

Figure 8: Coffee breaks with delicious Sicilian coffee.

H2020 project AHEAD funded by the European Union as Research and Innovation Action under Grants No: 654215 and 871158. 


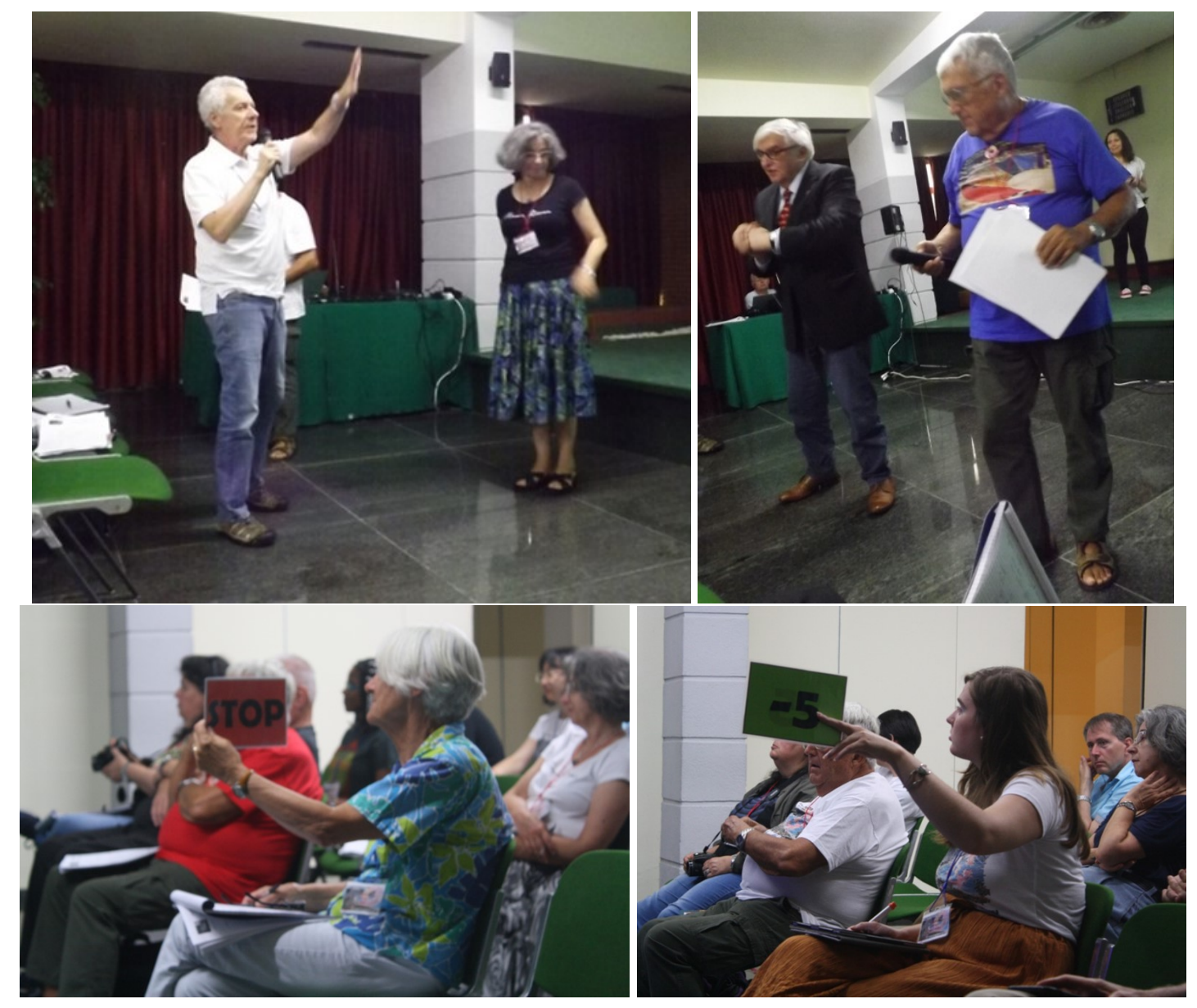

Figure 9: Conference chairs. 

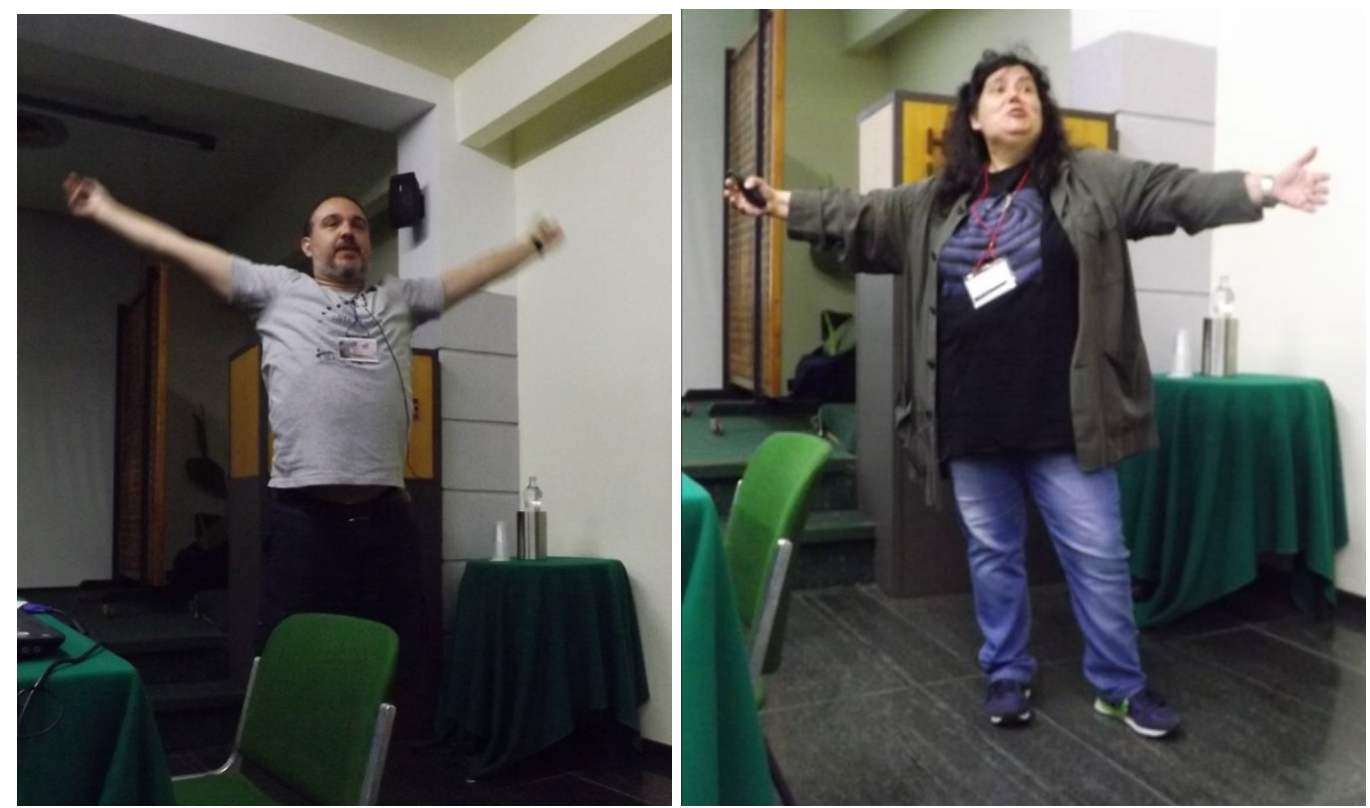

Figure 10: In this conference, quite numerous speakers have illustrated the size of accretion disc in their models, in some cases even in rotation (left).
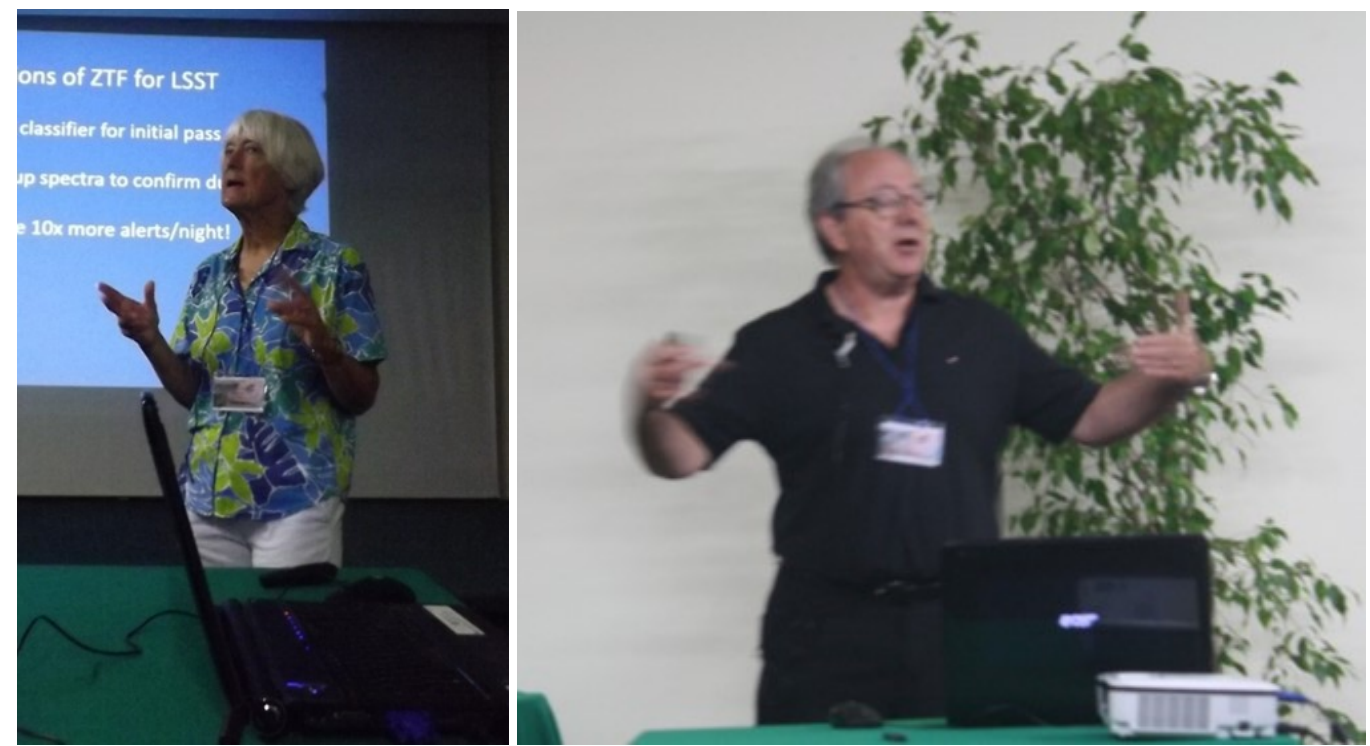

Figure 11: Another speakers demonstrating sizes of their accretion discs... 


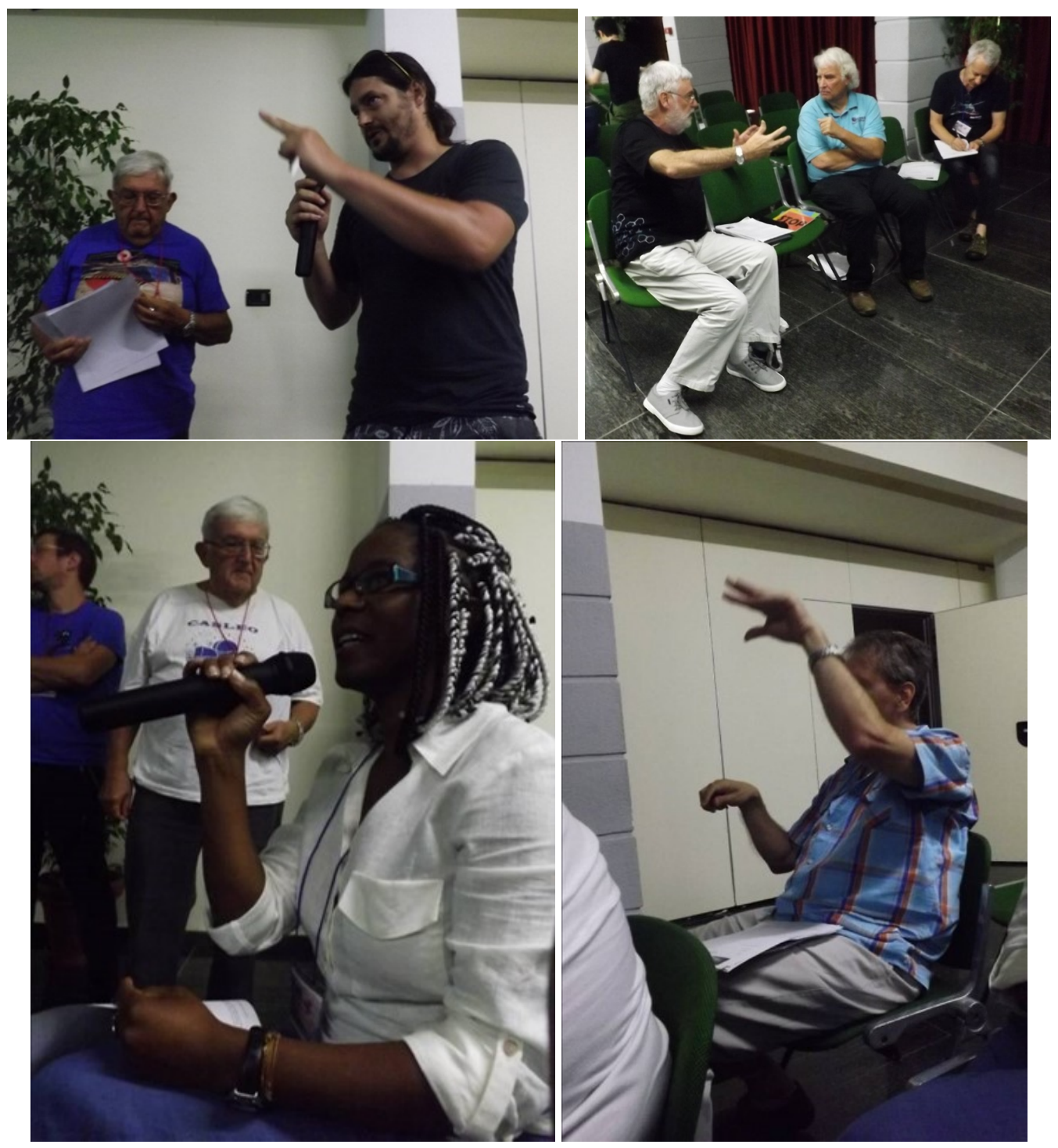

Figure 12: Questions/answers represented important part of the conference. 


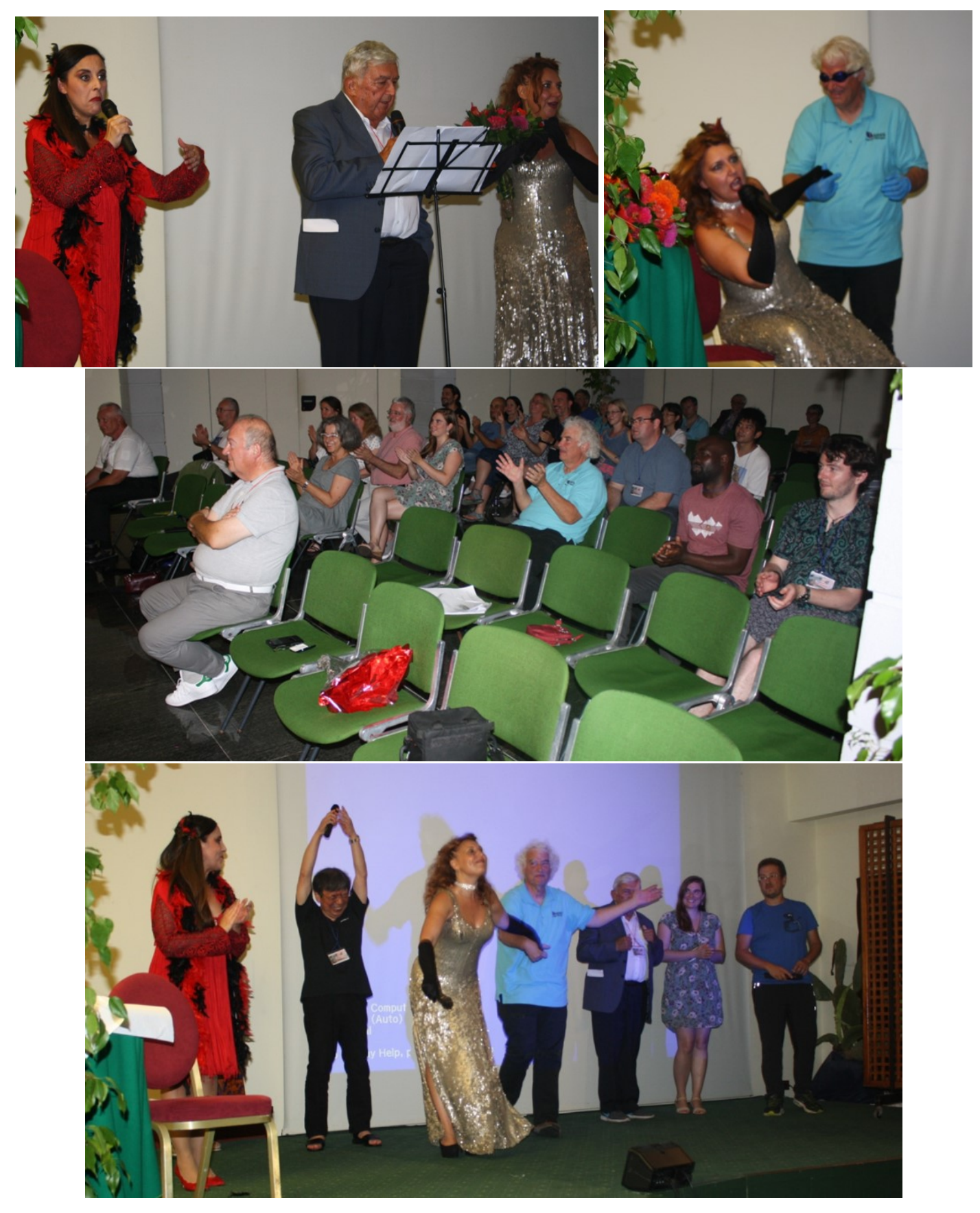

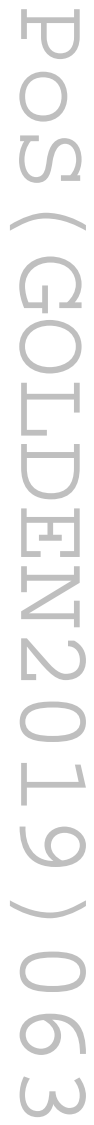

Figure 13: Cultural event. 

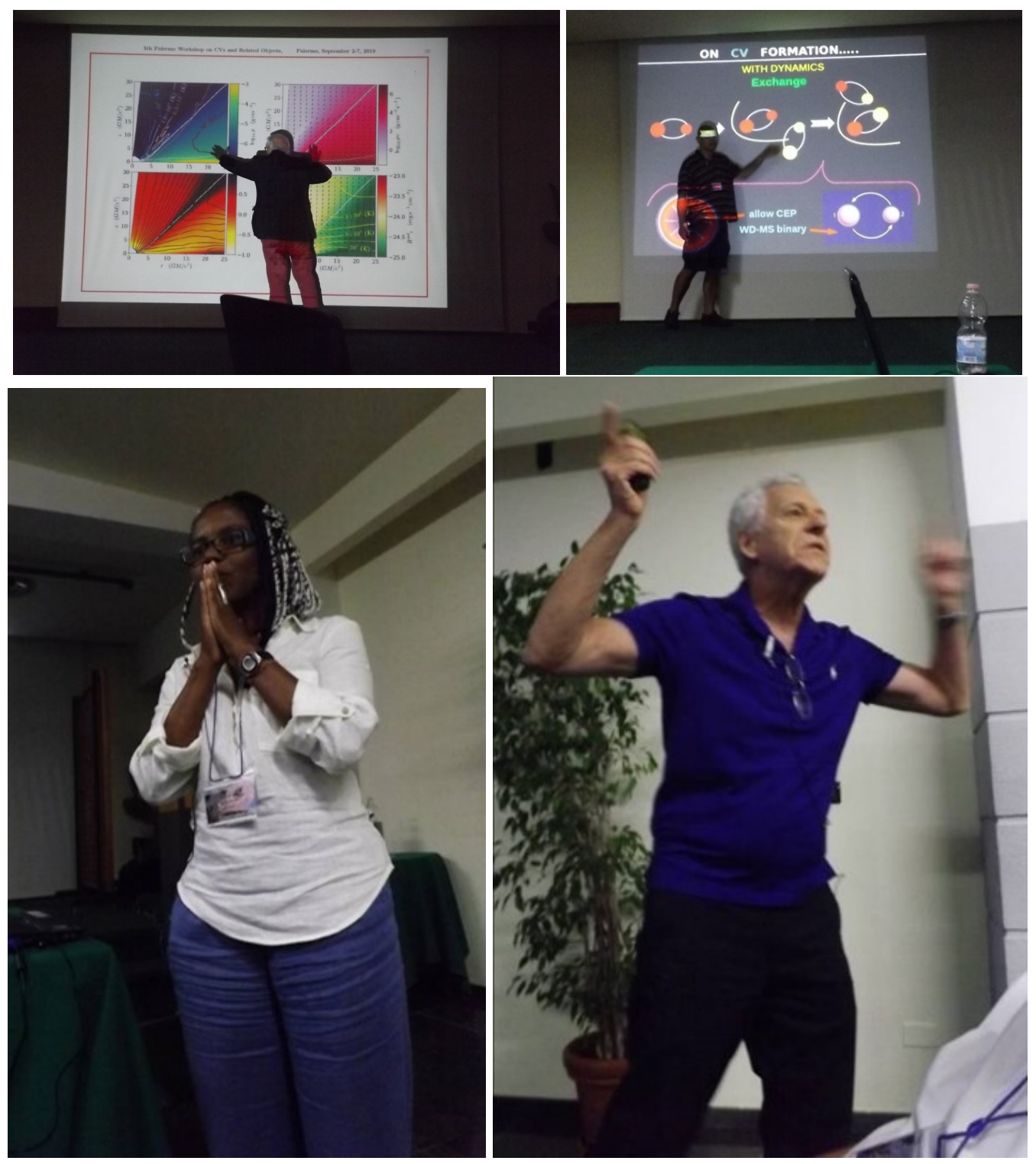

\begin{tabular}{l}
0 \\
0 \\
0 \\
0 \\
0 \\
0 \\
0 \\
0 \\
0 \\
\hline
\end{tabular}

Figure 14: Conference highlights. 


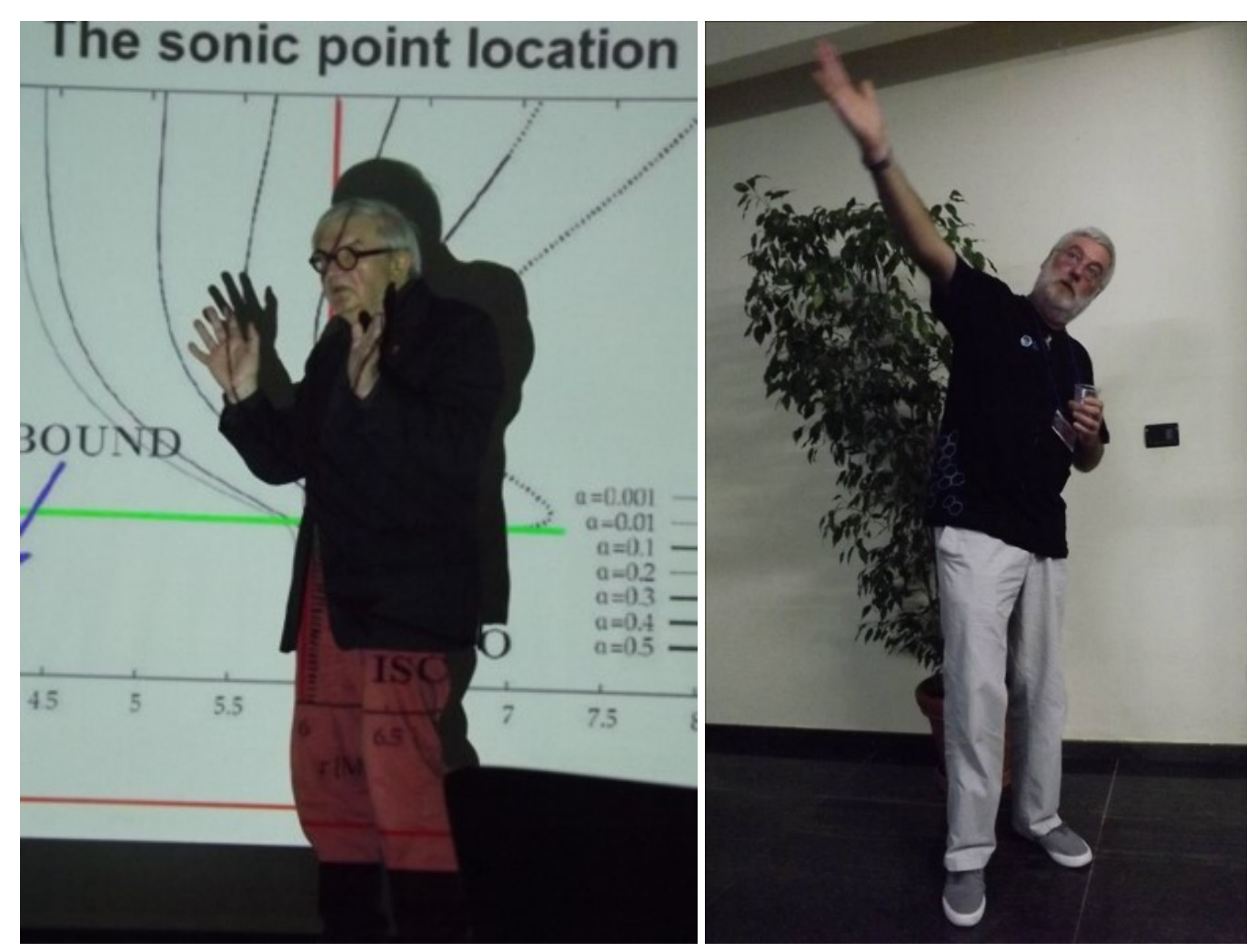

Figure 15: Conference highlights II. 